\title{
Familia, amigos y otras fuentes de información asociadas al inicio de las relaciones sexuales en adolescentes de El Salvador
}

\author{
Miguel Ruiz-Canela, ${ }^{1}$ Cristina López-del Burgo, ${ }^{1}$ Silvia Carlos, ${ }^{1}$ \\ María Calatrava, ${ }^{2}$ Alfonso Osorio $^{3}$ y Jokin de Irala ${ }^{2}$
}

Forma de citar

Ruiz-Canela M, López-del Burgo C, Carlos S, Calatrava M, Osorio A, de Irala J. Familia, amigos y otras fuentes de información asociadas al inicio de las relaciones sexuales en adolescentes de El Salvador. Rev Panam Salud Publica. 2012;31(1):54-61.

RESUMEN Objetivo. El objetivo de este estudio es comprobar cómo influyen en el inicio de la actividad sexual de los jóvenes salvadoreños los mensajes que reciben sobre cuestiones de sexualidad, afectividad y ocio a través de la familia, los amigos y los medios de comunicación.

Métodos. Estudio transversal a partir de una muestra representativa de 2615 estudiantes (de 13 a 19 años) de El Salvador. Se utilizó un muestreo sistemático aleatorio para seleccionar 30 colegios. Se recogieron aspectos sociodemográficos, estilos de vida y fuentes de información sobre sexualidad y amor utilizadas por los jóvenes.

Resultados. La edad media de los jóvenes fue de 15 años $(D E=1,8)$. En total $638(24,4 \%)$ jóvenes afirmaron haber tenido relaciones sexuales. Los siguientes factores se asociaron con una mayor probabilidad de haber tenido relaciones sexuales: percibir que los hermanos $(O R=$ 1,8, IC 95\%: 1,2-2,7) o los amigos (OR =1,7, IC 95\%: 1,3-2,2) apoyan que se tengan relaciones sexuales. Como factores protectores se encontraron la supervisión de los padres $(O R=0,5$, IC 95\%: 0,4-0,7); recibir mensajes que apoyan la abstinencia por parte de amigos $(O R=0,7$, IC 95\%: 0,6-1,0) o hermanos (OR =0,7, IC 95\%:0,5-0,8) y recibir mensajes favorables al matrimonio por parte de los padres (OR =0,4, IC 95\%:0,3-0,6).

Conclusiones. Los mensajes de la familia y amigos son factores que parecen influir en el inicio de las relaciones sexuales de los jóvenes. Los programas de promoción de la salud sexual en El Salvador deberían tener en cuenta estos factores.

Palabras clave Conducta sexual; conducta del adolescente; factores socioeconómicos; El Salvador.

El comienzo precoz de las relaciones sexuales se asocia con una mayor probabilidad de tener más parejas a lo largo

\footnotetext{
Universidad de Navarra, Facultad de Medicina, Departamento de Medicina Preventiva y Salud Pública, Pamplona, España. La correspondencia se debe enviar a Miguel Ruiz-Canela, mcanela@unav.es

2 Universidad de Navarra, Instituto Cultura y Sociedad, Pamplona, España.

3 Universidad de Navarra, Facultad de Filosofía y Letras, Departamento de Educación, Pamplona, España.
}

de la vida y esto a su vez incrementa el riesgo de embarazos e infecciones de transmisión sexual (ITS) en la población (1-5). Esto es un problema de salud pública compartido por casi todos los países. El Salvador es un país en el que $61 \%$ de la población tiene de 0 a 29 años (6), y donde las mujeres de 15 a 24 años suponen un $36 \%$ de las mujeres en edad fértil (15-49 años) (7). En un estudio se encontró que $50 \%$ de los estudiantes varones salvadoreños habían tenido re- laciones sexuales a los 13 años o antes (8). Esto hace que las relaciones sexuales tempranas se hayan convertido en uno de los retos de las políticas sanitarias en este país (9).

La promoción de la salud es una de las herramientas de la salud pública para facilitar al cambio de las actividades de riesgo entre los jóvenes (10). Una medida para prevenir tanto el $\mathrm{VIH} /$ sida como otras ITS y los embarazos entre adolescentes es retrasar la edad de inicio 
de las relaciones sexuales (11-13). En este contexto, es importante identificar los factores individuales que se asocian con dicho inicio. Se ha sugerido que un escaso aprovechamiento en la escuela, el consumo de alcohol, tabaco y otras drogas, así como determinadas actividades realizadas durante el tiempo de ocio aumentan la posibilidad de tener relaciones sexuales tempranas (14-16).

La identificación de los factores individuales, sin embargo, no es suficiente ya que es necesario valorar el entorno que rodea a los jóvenes (17-19). En países desarrollados se ha demostrado la influencia de los mensajes que reciben de la familia, los amigos, los medios de comunicación y el ambiente socioeconómico (20-24). Algunos autores han señalado la especial relevancia que tienen los padres con respecto al inicio de las relaciones sexuales de los jóvenes (25). Se ha demostrado que la supervisión de los padres $(26,27)$, la comunicación padreshijos (28) o el grado de aprobación de las relaciones sexuales de los hijos por parte de los padres (28-31) son importantes determinantes de sexualidad precoz.

En los países en vías de desarrollo también se ha reconocido la importancia de este enfoque multicausal $(16,27$, 32). En varios países africanos se ha demostrado el papel de la comunicación y supervisión de los padres $(16,33)$. Otros estudios han valorado la influencia de factores externos, como el nivel económico, la estructura familiar o el comportamiento de los amigos, sobre la actividad sexual de los estudiantes en Perú (34), México (35) y Chile (36). Sin embargo, en una revisión exhaustiva se comprobó que la mayoría de los estudios en países latinoamericanos se han centrado fundamentalmente en los factores individuales, como la edad, el nivel de estudios o el consumo de alcohol (14).

Los estudios realizados en El Salvador se han centrado en comprobar cómo determinados aspectos individuales $\mathrm{y}$ socioeconómicos se relacionan con diversos comportamientos de riesgo, entre los que se incluye la actividad sexual (8, 37). Teniendo en cuenta las diferencias socioculturales con países de otras regiones, el objetivo general de este estudio es conocer las fuentes de información sobre cuestiones de sexualidad y qué influencia tienen en los estudiantes de El Salvador a la hora de tener sus primeras relaciones sexuales. Específicamente, se pretende comprobar la influencia de la familia, los amigos y los medios de comunicación, al mismo tiempo que se tienen en cuenta otros factores individuales relacionados con el estilo de vida de los jóvenes salvadoreños.

\section{MATERIALES Y MÉTODOS}

Este estudio transversal forma parte de un proyecto internacional sobre la actividad y las actitudes de los jóvenes en materia de estilos de vida y sexualidad (Proyecto YOURLIFE) $(38,39)$.

\section{Participantes}

La encuesta se realizó en 2008 sobre una muestra representativa de la población adolescente de $7^{\circ}$ grado a $3^{\circ}$ de bachillerato de El Salvador, con edades comprendidas entre 13 y 19 años. Para ello se realizó un muestreo sistemático aleatorio para seleccionar 30 colegios representativos según cuatro criterios: grado de estudio, región geográfica, tipo de institución (pública/privada) y municipio donde se encuentra el colegio. La región geográfica estudiada se centró en los centros educativos localizados en tres áreas urbanas de El Salvador (San Salvador, Santa Ana y San Miguel) que suponen aproximadamente el $40 \%$ de la población estudiantil de este país.

Todos los estudiantes presentes en las aulas respondieron al cuestionario. De los 3259 cuestionarios recibidos, se excluyeron 644 (20\%). Entre ellos hubo 308 cuestionarios excluidos porque contenían datos incongruentes (por ejemplo, la edad de la primera relación sexual era mayor que la edad actual). Este número de datos incongruentes es inferior o similar al comunicado en estudios semejantes (40-42). El resto se excluyó porque no informaban sobre alguna de las variables principales del estudio. La muestra inicial se comparó con la muestra final según la distribución por las variables sociodemográficas (sexo, edad, nivel económico, medio en el que viven, tipo de colegio y si habían tenido relaciones sexuales) y no se encontraron diferencias significativas.

\section{Cuestionario}

Se elaboró un cuestionario estructurado con 59 preguntas. Las respuestas debían ser marcadas con un lápiz por los jóvenes. Este cuestionario se adaptó a partir de una versión en inglés que fue aplicada previamente en Filipinas (38).

Aparte del estudio piloto realizado con 180 jóvenes filipinos con la versión inglesa del cuestionario, se efectuó una segunda prueba piloto del cuestionario en español adaptado para El Salvador en dos grupos focales de 10 jóvenes cada uno (10 varones y 10 mujeres, de colegios privados y públicos de San Salvador, de nivel socioeconómico mediobajo). Estos estudios previos permitieron tener en cuenta los factores cognitivos que influyen en la validez de los cuestionarios autoadministrados dirigidos a los adolescentes (43). En concreto, se mejoró la redacción de algunas preguntas y la presentación formal de otras, de modo que los jóvenes comprendieran el cuestionario y supieran responder adecuadamente.

El cuestionario recogía las siguientes variables: a) relaciones sexuales: si habían tenido relaciones sexuales y a qué edad tuvieron la primera; b) aspectos sociodemográficos: sexo, edad, nivel económico, medio en el que viven (urbano, rural), si viven con los padres y tipo de colegio (público o privado); c) nivel de religiosidad: frecuencia con la que acudían a la iglesia o templo y si estaban de acuerdo en que la fe supone una influencia importante para su vida; d) responsabilidad personal: si eran personas que solían terminar bien sus proyectos y que planificaban su futuro; e) frecuencia de uso de fuentes de información sobre temas relacionados con el amor y la sexualidad (padre, madre, hermanos, amigos, profesor, sacerdote, revistas/Internet o libros); f) mensajes transmitidos por padres, profesores, televisión, cine o revistas sobre la solidaridad, la lealtad o el respeto a las personas mayores; la sinceridad, la personalidad y la voluntad; y mensajes positivos sobre el matrimonio; g) fuentes de su entorno (padres, hermanos, amigos, profesores, medios de comunicación) que estarían de acuerdo con las frases "me parece bien que los jóvenes de tu edad tengan relaciones sexuales para pasárselo bien, sin que haya amor ni compromiso" y "es mejor esperar al compromiso del matrimonio (casarse), para tener relaciones sexuales"; h) cuestiones tratadas con los padres: frecuencia con la que hablaban sobre aspectos de biología de la sexualidad y la reproducción; el ena- 
moramiento; las relaciones sexuales; la integración social y el consumo de drogas y alcohol; y sobre la prevención de ITS y el uso de anticonceptivos; i) importancia concedida a la opinión de padres y amigos. Se plantearon 8 cuestiones: manera de vestir, hora de volver a casa, fumar y beber alcohol, consumir drogas ilegales, actividades en el tiempo libre, temas de amor y sexualidad, temas de religión y sobre la elección de los amigos y amigas; j) grado de supervisión de los padres sobre las actividades que realizan durante el tiempo libre; k) estilo de vida de los jóvenes: frecuencia de consumo de tabaco, alcohol o de otras drogas (marihuana, hachís, etc.); frecuencia semanal de 16 actividades realizadas durante su tiempo libre, como ir a discotecas y bares, reunirse en locales sin adultos, estar en salas de juegos, ver programas culturales en televisión o leer libros; y dinero gastado al mes para realizar distintas actividades relacionadas con su ocio, incluyendo las nuevas tecnologías.

Las respuestas en todas estas preguntas de frecuencia fueron de tipo Likert con una puntuación mínima de 1 y una máxima de 5 .

\section{Análisis estadístico}

Se utilizó el test exacto de Fisher de dos colas o el test de la ji al cuadrado para comparar las frecuencias entre grupos. Posteriormente se realizó un análisis de regresión logística multivariante no condicional donde la variable dependiente era si los jóvenes habían mantenido o no relaciones sexuales alguna vez en su vida. Las variables independientes incluidas fueron las que recogían la posible influencia de los factores externos en la primera actividad sexual de los jóvenes: fuentes de información utilizadas habitualmente (casi siempre o siempre) para conocer aspectos sobre el amor y la sexualidad; fuentes que transmitían valores relacionados con la solidaridad, aspectos de la personalidad o el matrimonio; considerar importante la opinión de padres y amigos sobre su estilos de vida (cuartil más bajo frente al resto); hablar frecuentemente (bastante o mucho) con los padres sobre al menos uno de los temas descritos previamente sobre sexualidad, ITS y prevención, afectividad y amor, tipos y lugar de diversión y temas culturales; fuentes de su entorno que consideran el sexo como un modo de diversión para los jóvenes o que apoyan la abstinencia; vivir con los padres; supervisión alta de los padres con respecto a las actividades desarrolladas durante el tiempo libre.

Como posibles variables de confusión también se introdujeron en el modelo multivariante todas las cuestiones sociodemográficas y de estilos de vida. Con respecto a las actividades realizadas durante el tiempo libre se incluyeron dos variables continuas, una que sumaba las actividades consideradas de riesgo (ir a discotecas, frecuentar lugares sin adultos, etc.) y otra que sumaba las actividades culturales. En relación con el consumo de tabaco, alcohol u otro tipo de drogas, se comparó a los jóvenes que no habían consumido nunca ninguna de estas sustancias frente al resto.

\section{Aspectos éticos}

El cuestionario fue aprobado por el Comité de Ética de Investigación de la Universidad de Málaga. Antes de realizar la encuesta se obtuvo la aprobación del comité directivo de cada colegio, que en cada caso era el responsable de informar y buscar la aprobación de los padres. Al principio del cuestionario se incluía una breve explicación del estudio y se explicaba el carácter voluntario y anónimo de la encuesta; también se recalcaba su libertad para dejar sin respuesta cualquier pregunta del cuestionario. De este modo, responder el cuestionario implicaba el consentimiento para participar en el estudio.

\section{RESULTADOS}

El estudio incluyó 2615 jóvenes con una edad media de 15 años ( $D E=1,8$ ), de los cuales 1468 eran varones (56\%) y 1147 mujeres (44\%). En total, 638 jóvenes $(24,4 \%)$ respondieron haber tenido relaciones sexuales alguna vez en su vida. La relación entre las variables sociodemográficas y haber tenido relaciones sexuales se muestra en el cuadro 1. En todos los casos, excepto con el tipo de colegio (público o privado), la asociación con haber tenido relaciones sexuales fue estadísticamente significativa. Se observa que la frecuencia de relaciones sexuales fue significativamente mayor en los varones, los estudiantes de mayor

CUADRO 1. Características sociodemográficas y comportamiento sexual de una muestra representativa de jóvenes estudiantes de El Salvador, 2008

\begin{tabular}{|c|c|c|c|}
\hline \multirow[b]{2}{*}{ Características $(n)$} & \multicolumn{2}{|c|}{ Inicio de las relaciones sexuales } & \multirow[b]{2}{*}{ Valor $P$} \\
\hline & No. & $\%$ & \\
\hline \multicolumn{4}{|l|}{ Sexo } \\
\hline Hombre (1 468) & 448 & 30,5 & $<0,001$ \\
\hline Mujer (1 147) & 190 & 16,6 & \\
\hline \multicolumn{4}{|l|}{ Edad (años) } \\
\hline $13(447)$ & 35 & 7,8 & $<0,001$ \\
\hline $14(519)$ & 83 & 16,0 & \\
\hline $15(494)$ & 108 & 21,9 & \\
\hline $16(403)$ & 120 & 29,8 & \\
\hline $17(380)$ & 131 & 34,5 & \\
\hline $18(258)$ & 104 & 40,3 & \\
\hline $19(114)$ & 57 & 50,0 & \\
\hline \multicolumn{4}{|l|}{ Nivel económico } \\
\hline Bajo (431) & 121 & 28,1 & 0,003 \\
\hline Medio (1 777) & 399 & 22,5 & \\
\hline Alto (407) & 118 & 29,0 & \\
\hline \multicolumn{4}{|l|}{ Medio en el que vive } \\
\hline Rural (272) & 52 & 19,1 & 0,035 \\
\hline Urbano (1 906) & 464 & 24,4 & \\
\hline No sabe/no contesta (437) & 121 & 27,7 & \\
\hline \multicolumn{4}{|l|}{ Religiosidad $^{\mathrm{b}}$} \\
\hline Alta (1 305) & 241 & 18,5 & $<0,001$ \\
\hline Baja o ninguna (1 310) & 397 & 30,3 & \\
\hline \multicolumn{4}{|l|}{ Tipo de colegio } \\
\hline Privado (1 013) & 263 & 26,0 & 0,147 \\
\hline Público (1 602) & 375 & 23,4 & \\
\hline
\end{tabular}


edad, aquellos con un nivel económico más alto, los que viven en un medio urbano y los que no tienen un nivel alto de religiosidad.

La principal fuente de información utilizada por los estudiantes para tratar de forma frecuente (siempre o casi siempre) aspectos relacionados con el amor y la sexualidad fueron los padres (37\%), seguidos de los amigos (33\%), los hermanos $(17 \%)$, los libros (14\%), las revistas o Internet (13\%), el profesor $(10 \%)$ y el sacerdote $(6 \%)$. En el cuadro 2 se muestra la proporción de estudiantes que habían tenido relaciones sexuales según las fuentes de información utilizadas con mayor frecuencia. El porcentaje de jóvenes con relaciones sexuales era mayor entre los que utilizaron como fuentes de información a los amigos o revistas/Internet si se compara con los jóvenes que no utilizaron estas fuentes. Se encontró un porcentaje estadísticamente mayor de jóvenes con relaciones sexuales entre los que no utilizaban como fuente de información a los padres. No se encontraron diferencias estadísticamente significativas cuando la fuente habitual de información eran los hermanos o un profesor.

En el cuadro 3 se recoge el porcentaje de inicio en las relaciones sexuales según la exposición a dos mensajes procedentes de distintas fuentes de información. Se observa que la proporción de jóvenes que habían tenido relaciones sexuales fue mayor en aquellos que recibían mensajes que aprueban el sexo como modo de diversión. Esta asociación fue estadísticamente significativa en todas las fuentes de información excepto para los medios de comunicación. La frecuencia de relaciones fue inferior entre los que afirmaron recibir mensajes que apoyaban el retraso de la edad de inicio de las relaciones sexuales frente a los que no recibían estos mensajes por distintas fuentes. Igualmente las diferencias fueron siempre estadísticamente significativas excepto para los medios de comunicación.

En el cuadro 4 se presenta la asociación entre haber tenido relaciones sexuales, aspectos relativos a la relación que mantenían con los padres y diversas cuestiones tratadas con los padres. En estos resultados se observa que la frecuencia de haber tenido relaciones sexuales era menor en aquellos que frecuentemente habían tratado con los padres cuestiones sobre el amor y el enamoramiento, así
CUADRO 2. Inicio de las relaciones sexuales en una muestra representativa de jóvenes estudiantes de El Salvador (13-19 años) según la fuente de información utilizada para aprender sobre temas de amor y sexualidad, 2008

\begin{tabular}{|c|c|c|c|}
\hline \multirow[b]{2}{*}{ Fuente de información utilizada $(n)$} & \multicolumn{2}{|c|}{ Inicio de las relaciones sexuales } & \multirow[b]{2}{*}{ Valor $P a$} \\
\hline & No. & $\%$ & \\
\hline \multicolumn{4}{|l|}{ Padres $^{b}$} \\
\hline Siempre o casi siempre (963) & 183 & 19,0 & $<0,001$ \\
\hline Resto (1 652) & 455 & 27,5 & \\
\hline \multicolumn{4}{|l|}{ Amigos } \\
\hline Siempre o casi siempre (874) & 284 & 32,5 & $<0,001$ \\
\hline No $(1741)$ & 354 & 20,3 & \\
\hline \multicolumn{4}{|l|}{ Hermanos } \\
\hline Siempre o casi siempre (441) & 121 & 27,4 & 0,114 \\
\hline No $(2$ 174) & 517 & 23,8 & \\
\hline \multicolumn{4}{|l|}{ Revistas e Internet } \\
\hline Siempre o casi siempre (327) & 103 & 31,5 & 0,002 \\
\hline No (2 288) & 535 & 23,4 & \\
\hline \multicolumn{4}{|l|}{ Profesor } \\
\hline Siempre o casi siempre (253) & 65 & 25,7 & 0,644 \\
\hline No $(2362)$ & 573 & 24,3 & \\
\hline
\end{tabular}

a Prueba exacta de Fisher de dos colas.

b La madre, el padre o ambos.

CUADRO 3. Inicio de las relaciones sexuales en una muestra representativa de jóvenes estudiantes de El Salvador (13-19 años) según los mensajes recibidos desde diversas fuentes de información, 2008

\begin{tabular}{|c|c|c|c|}
\hline \multirow[b]{2}{*}{ Mensaje recibido $(n)$} & \multicolumn{2}{|c|}{ Inicio de las relaciones sexuales } & \multirow[b]{2}{*}{ Valor $P^{a}$} \\
\hline & No. & $\%$ & \\
\hline $\begin{array}{l}\text { El sexo en los jóvenes como diversión e } \\
\text { apoyada por: }\end{array}$ & & & \\
\hline $\begin{array}{l}\text { Padres } \\
\text { Nob }(2470) \\
\text { Sí (144) }\end{array}$ & $\begin{array}{r}582 \\
55\end{array}$ & $\begin{array}{l}23,6 \\
38,2\end{array}$ & $<0,001$ \\
\hline $\begin{array}{l}\text { Amigos } \\
\text { No }{ }^{\mathrm{b}}(2 \text { 161) } \\
\text { Sí (453) }\end{array}$ & $\begin{array}{l}438 \\
199\end{array}$ & $\begin{array}{l}20,3 \\
43,9\end{array}$ & $<0,001$ \\
\hline $\begin{array}{l}\text { Hermanos } \\
\text { No }{ }^{b}(2453) \\
\text { Sí (161) }\end{array}$ & $\begin{array}{r}558 \\
79\end{array}$ & $\begin{array}{l}22,7 \\
49,1\end{array}$ & $<0,001$ \\
\hline $\begin{array}{l}\text { Profesor } \\
\text { Nob }(2495) \\
\text { Sí (119) }\end{array}$ & $\begin{array}{r}588 \\
49\end{array}$ & $\begin{array}{l}23,6 \\
41,2\end{array}$ & $<0,001$ \\
\hline $\begin{array}{l}\text { Medios de comunicación } \\
\text { Nob }(2 \text { 019) } \\
\text { Sí (595) }\end{array}$ & $\begin{array}{l}489 \\
148\end{array}$ & $\begin{array}{l}24,2 \\
24,9\end{array}$ & 0,745 \\
\hline La abstinencia es una opinión apoyada & & & \\
\hline $\begin{array}{l}\text { Padres } \\
\text { No }{ }^{\mathrm{b}}(536) \\
\text { Sí }(2078)\end{array}$ & $\begin{array}{l}178 \\
459\end{array}$ & $\begin{array}{l}33,2 \\
22,1\end{array}$ & $<0,001$ \\
\hline $\begin{array}{l}\text { Amigos } \\
\text { Nob }(1690) \\
\text { Sí (924) }\end{array}$ & $\begin{array}{l}505 \\
132\end{array}$ & $\begin{array}{l}29,9 \\
14,3\end{array}$ & $<0,001$ \\
\hline $\begin{array}{l}\text { Hermanos } \\
\text { No }{ }^{\mathrm{b}}\left(\begin{array}{l}1 \\
265)\end{array}\right. \\
\text { Sí (1 } 349)\end{array}$ & $\begin{array}{l}415 \\
222\end{array}$ & $\begin{array}{l}32,8 \\
16,5\end{array}$ & $<0,001$ \\
\hline $\begin{array}{l}\text { Profesor } \\
\text { Nob }(1115) \\
\text { Sí (1 499) }\end{array}$ & $\begin{array}{l}340 \\
297\end{array}$ & $\begin{array}{l}30,5 \\
19,8\end{array}$ & $<0,001$ \\
\hline $\begin{array}{l}\text { Medios de comunicación } \\
\text { No }{ }^{\text {b }}(2030) \\
\text { Sí }(584)\end{array}$ & $\begin{array}{l}509 \\
128\end{array}$ & $\begin{array}{l}25,1 \\
21,9\end{array}$ & 0,126 \\
\hline
\end{tabular}

a Prueba exacta de Fisher de dos colas.

b Se incluyen tanto los que no lo indicaron como los valores perdidos. 
CUADRO 4. Inicio de las relaciones sexuales en una muestra representativa de jóvenes estudiantes de El Salvador (13-19 años) según la relación mantenida con sus padres, 2008

\begin{tabular}{|c|c|c|c|}
\hline \multirow[b]{2}{*}{ Relacion con los padres $(n)$} & \multicolumn{2}{|c|}{ Inicio de las relaciones sexuales } & \multirow[b]{2}{*}{ Valor $P^{a}$} \\
\hline & No. & $\%$ & \\
\hline \multicolumn{4}{|l|}{ Temas tratados frecuentemente con los padres ${ }^{b}$} \\
\hline \multicolumn{4}{|l|}{ Amor y enamoramiento ${ }^{C}$} \\
\hline $\mathrm{No}^{\mathrm{d}}(1574)$ & 420 & 26,7 & 0,001 \\
\hline Sí (1 041) & 218 & 20,9 & \\
\hline \multicolumn{4}{|l|}{ Relaciones sexuales ${ }^{e}$} \\
\hline $\mathrm{No}^{\mathrm{d}}(1679)$ & 428 & 25,5 & 0,087 \\
\hline Sí (936) & 210 & 22,4 & \\
\hline \multicolumn{4}{|l|}{ Cuestiones biológicas de sexualidad ${ }^{f}$} \\
\hline $\mathrm{No}^{\mathrm{d}}(1 \mathrm{109})$ & 308 & 27,8 & 0,001 \\
\hline Sí (1 506) & 330 & 21,9 & \\
\hline \multicolumn{4}{|l|}{ Uso de anticonceptivos o prevención de ITS } \\
\hline No $^{d}(1291)$ & 316 & 24,5 & 0,928 \\
\hline Sí (1 324) & 322 & 24,3 & \\
\hline \multicolumn{4}{|l|}{ Actividades de ociog } \\
\hline $\mathrm{No}^{\mathrm{d}}(847)$ & 222 & 26,2 & 0,144 \\
\hline Sí (1 768) & 416 & 23,5 & \\
\hline \multicolumn{4}{|l|}{ Supervisión de los padres ${ }^{h}$} \\
\hline Bastante/mucho (1 699) & 301 & 17,7 & $<0,001$ \\
\hline Poco/nada (898) & 334 & 37,2 & \\
\hline \multicolumn{4}{|l|}{ Vive con padre y madre } \\
\hline Sí (1 540) & 337 & 21,9 & $<0,001$ \\
\hline Resto $^{d}\left(\begin{array}{ll}1 & 075)\end{array}\right.$ & 300 & 28,0 & \\
\hline \multicolumn{4}{|c|}{$\begin{array}{l}\text { Los padres transmiten mensajes positivos sobre } \\
\text { el matrimonio }\end{array}$} \\
\hline Sí (2 367) & 535 & 22,6 & $<0,001$ \\
\hline 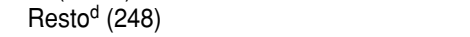 & 103 & 41,5 & \\
\hline \multicolumn{4}{|c|}{$\begin{array}{l}\text { Importancia de la opinión de los padres sobre sus } \\
\text { estilos de vida y actividad social }\end{array}$} \\
\hline $\operatorname{Alta}^{\mathrm{j}}(2$ 147) & 472 & 22,0 & $<0,001$ \\
\hline Resto (468) & 166 & 35,5 & \\
\hline \multicolumn{4}{|l|}{ Los padres le dan todo lo que les piden } \\
\hline Casi siempre/siempre (691) & 194 & 28,1 & 0,010 \\
\hline Restod $^{d}(1924)$ & 444 & 23,1 & \\
\hline
\end{tabular}

ITS: infecciones de transmisión sexual.

a Prueba exacta de Fisher de dos colas.

b Se considera positivo cuando al menos trataba frecuentemente una cuestión relacionada con el tema.

c Cómo saber cuándo se está preparado para salir con una chica, o qué significa enamorarse.

d Se incluyen tanto los que no lo indicaron como los valores perdidos.

e Cómo saber cuándo estar preparado para tener relaciones sexuales, cómo manejar el impulso sexual o cómo distinguir entre deseo, atracción sexual y amor

f Cambios en el cuerpo de las chicas y los chicos, o información sobre el embarazo y el comienzo de la vida.

${ }^{9}$ Consumo de drogas y alcohol, lugares de diversión, participación en pandillas, la prostitución y la pornografía.

${ }^{\mathrm{h}}$ Frecuencia con la que los padres saben qué hacen los jóvenes durante su tiempo libre (18 jóvenes no respondieron a esta cuestión).

Incluye "acertar en la elección de la pareja con quien formar una familia" o "si te casas, que el matrimonio sea para toda la vida".

j Incluye a los jóvenes que sí concedían importancia a la opinión de sus padres sobre un número importante de cuestiones sobre sus estilos de vida y actividad social (al menos 3 de un total de 8 ).

como sobre temas de biología sexual, como los cambios sexuales que experimentan los jóvenes durante la adolescencia $(P=0,001$ en ambos casos).

Además de lo indicado en el cuadro 4, también se encontraron diferencias al comparar los que consideraban importante la opinión de sus amigos en al menos un tema relacionado con su estilo de vida (como fumar, consumir drogas, actividades durante el tiempo libre o la hora de volver a casa) frente al resto. Entre los que contestaron que esta opinión era importante, 20,5\% había mantenido relaciones sexuales frente a $26,9 \%$ entre los que señalaron que esta opinión no era importante $(P=0,001)$. También se encontraron diferencias relacionadas con la capacidad expresada por los estudiantes para terminar bien los proyectos que empiezan: $22,8 \%$ de los que respondieron afirmativamente a esta pregunta tuvieron relaciones sexuales, frente a $29,3 \%$ de los que señalaron que no $(P=0,001)$. $Y$, del mismo modo, hubo diferencias en las relaciones sexua- les entre los que afirmaron que suelen pensar y planificar su futuro $(22,6 \%)$ y los que no $(30,4 \%, P<0,001)$.

En el análisis multivariante (cuadro 5) se observa que una mayor supervisión parental, la transmisión de mensajes positivos sobre el matrimonio por parte de los padres, y tener amigos o hermanos que apoyen la abstinencia hasta el matrimonio son factores asociados con una menor frecuencia de tener relaciones sexuales. Por lo contrario, la frecuencia de haber tenido relaciones sexuales aumenta entre los estudiantes que referían recibir mensajes de amigos y hermanos considerando el sexo desde una perspectiva más lúdica. Estos factores se ajustaron con variables sociodemográficas y otras variables relacionadas con estilos de vida de los jóvenes.

\section{DISCUSIÓN}

Los resultados de esta encuesta han mostrado que las fuentes de información y los mensajes que reciben los jóvenes se asocian con el inicio de sus relaciones sexuales. Esta asociación se mantuvo en el análisis multivariante, por lo que puede afirmarse que determinados mensajes y fuentes de información pueden estar influyendo en los estudiantes salvadoreños independientemente del sexo, la edad, la religiosidad, el nivel económico y otras características asociadas con los estilos de vida como el consumo de drogas, tabaco y alcohol. Específicamente, parece que ejerce una gran influencia la percepción que tienen los estudiantes sobre los mensajes y la actitud de padres, hermanos y amigos sobre cuestiones relacionadas con la sexualidad.

Los resultados obtenidos confirman los hallazgos de estudios anteriores. La relación entre una mayor supervisión de los padres y el retraso en las relaciones sexuales coincide con estudios llevados a cabo en Estados Unidos (26, $30)$, en varios países africanos $(16,44)$ y en El Salvador (37). En investigaciones previas también se ha demostrado la influencia de la comunicación que los jóvenes establezcan con los padres (25, 28, 29, 31, 33, 45-47). Del mismo modo, e independientemente de esta influencia de los padres, se confirma que la opinión de los amigos y hermanos sobre el sexo también influye en el inicio de las relaciones sexuales en los jóvenes (24, 33, 35). No obstante, en un estudio reciente 
CUADRO 5. Odds ratios (razón de probabilidades) ajustadas para el inicio de las relaciones sexuales en una muestra representativa de jóvenes estudiantes de El Salvador (13-19 años), ${ }^{a}$ 2008

\begin{tabular}{|c|c|c|c|}
\hline \multirow[b]{2}{*}{ Factor asociado $(n)$} & \multicolumn{2}{|c|}{$\begin{array}{l}\text { Inicio de las relaciones } \\
\text { sexuales }^{\text {b }}\end{array}$} & \multirow[b]{2}{*}{ OR ajustada (IC95\%) } \\
\hline & No. & $\%$ & \\
\hline \multicolumn{4}{|l|}{ Supervisión de los padres ${ }^{c}$} \\
\hline Poco/nada (898) & 334 & 37,2 & 1,00 (ref.) $^{e}$ \\
\hline Bastante/mucho (1 699) & 301 & 17,7 & $0,5(0,4-0,7)$ \\
\hline \multicolumn{4}{|c|}{$\begin{array}{l}\text { Los padres transmiten mensajes positivos sobre } \\
\text { el matrimoniod }\end{array}$} \\
\hline No $(241)$ & 101 & 41,9 & 1,00 (ref.) \\
\hline Sí (2 356) & 534 & 22,7 & $0,4(0,3-0,6)$ \\
\hline \multicolumn{4}{|l|}{ La abstinencia es apoyada por los amigos } \\
\hline No (1 673) & 502 & 30,0 & 1,00 (ref.) \\
\hline Sí (924) & 133 & 14,4 & $0,7(0,6-1,0)$ \\
\hline \multicolumn{4}{|c|}{ La abstinencia es apoyada por los hermanos } \\
\hline No (1 252) & 412 & 32,9 & 1,00 (ref.) \\
\hline Sí (1 345) & 223 & 16,6 & $0,7(0,5-0,8)$ \\
\hline \multicolumn{4}{|c|}{$\begin{array}{l}\text { El sexo en los jóvenes como diversión apoyado por } \\
\text { los amigos }\end{array}$} \\
\hline No (2 147) & 436 & 20,3 & 1,00 (ref.) \\
\hline Sí (450) & 199 & 44,2 & $1,7(1,3-2,2)$ \\
\hline \multicolumn{4}{|c|}{$\begin{array}{l}\text { El sexo en los jóvenes como diversión apoyado por } \\
\text { los hermanos }\end{array}$} \\
\hline No $(2436)$ & 556 & 22,8 & 1,00 (ref.) \\
\hline Sí (161) & 79 & 49,1 & $1,8(1,2-2,7)$ \\
\hline \multicolumn{4}{|c|}{$\begin{array}{l}\text { a Modelo de regresión logística ajustado también por sexo, edad, nivel económico, religiosidad, consumo de tabaco/alcohol/ } \\
\text { drogas, otras conductas de riesgo durante el tiempo de ocio (como acudir a una discoteca, o reunirse en locales sin adultos) } \\
\text { y hábitos positivos relacionados con actividades culturales o de estudio. } \\
\text { b En el análisis se incluyó sólo a } 2597 \text { jóvenes porque } 18 \text { no informaron sobre la supervisión de sus padres. } \\
\text { c Frecuencia con la que los padres saben qué hacen los jóvenes durante su tiempo libre. } \\
\text { d Incluye "acertar en la elección de la pareja con quien formar una familia" o "si te casas, que el matrimonio sea para toda la } \\
\text { vida". } \\
\text { e ref = categoría utilizada como referencia para el cálculo de la OR. }\end{array}$} \\
\hline
\end{tabular}

se encontró que no había asociación entre el efecto protector de los factores familiares y parentales y el inicio de las relaciones sexuales cuando se ajusta por los problemas de salud mental (síntomas de depresión o ansiedad) y de comportamiento de los jóvenes (36).

La principal limitación de este estudio es su diseño transversal, ya que en algunas de las asociaciones encontradas no es fácil descartar una causalidad inversa. Por ejemplo, puede ocurrir que los padres empiecen a hablar con mayor frecuencia sobre los riesgos de las ITS y el uso de anticonceptivos cuando conocen o sospechan que sus hijos mantienen relaciones sexuales. No obstante, la causalidad inversa no parece una explicación plausible para todas las asociaciones encontradas en el análisis multivariante. No parece tan admisible, por ejemplo, que la postura de los padres, hermanos o amigos ante el sexo sea consecuencia del hecho de que los jóvenes hayan mantenido relaciones sexuales. Y algo similar puede afirmarse con lo que refieren los jóvenes sobre la supervisión de las mujeres salvadoreñas de 15 a 19 años vive en unión conyugal (7). No obstante, según la misma encuesta, la mayor parte de las mujeres que tuvieron su primera relación sexual entre los 15 y 19 años eran solteras. Teniendo en cuenta que el porcentaje de mujeres casadas es mayor en las que tienen 19 años, se realizó un nuevo análisis multivariante excluyendo el grupo de jóvenes con esta edad. Los resultados del análisis fueron prácticamente los mismos en cuanto a las variables asociadas y el valor de las odds ratios (razón de probabilidades) obtenidas.

La fortaleza de este estudio radica en que, por tratarse de una muestra representativa de estudiantes, los resultados pueden hacerse extensibles al resto de los jóvenes estudiantes de El Salvador. También es importante el tamaño de muestra utilizado ya que asegura la validez interna de los análisis y permite ajustar adecuadamente por los factores de confusión que están asociados con haber tenido relaciones sexuales entre los jóvenes. En este sentido, es relevante el hecho de haber valorado conjuntamente distintos factores que forman parte del entorno familiar y social que puede influir en el comportamiento de los jóvenes. Esto permite confirmar que es acertado reconocer el papel de los padres en los programas que promueven la salud de los jóvenes en países en vías de desarrollo (50). También es importante para recordar que la eficacia de estos programas puede estar determinada por la influencia de otros factores, como el entorno inmediato de los jóvenes.

En conclusión, este estudio muestra que el ambiente que rodea a los estudiantes, formado por la familia, los amigos y los medios de comunicación es un factor que influye en la actividad sexual de los jóvenes salvadoreños. En concreto, independientemente de las características sociodemográficas y de los estilos de vida, la frecuencia de jóvenes que han tenido relaciones sexuales es menor cuando los padres se preocupan de lo que hacen durante su tiempo libre y cuando los padres, amigos y hermanos transmiten mensajes que apoyan el retraso del inicio de las relaciones sexuales. Por el contrario, la frecuencia de jóvenes que han tenido relaciones sexuales aumenta en aquellos que piensan que tanto sus amigos como sus hermanos consideran al sexo como un modo de pasarlo bien, aunque no haya amor o 
compromiso. La identificación de estos factores refuerza la necesidad de un enfoque más global en los programas de promoción de la salud sexual. Específicamente, en los programas dirigidos a retrasar la edad de inicio de las relaciones sexuales y evitar la multiplicidad de parejas se debería tener en cuenta, no sólo los conocimientos y actitudes de los jóvenes salvadoreños sino también sus entornos próximos, que pueden ser contrarios a estos mensajes de evitación de riesgos.

Agradecimientos. Los autores agradecen a InterMedia Consulting (Italia), por haber impulsado este proyecto de investigación; al Consejo Nacional para la Cultura y el Arte de El Salvador (CONCULTURA), por la financiación del proyecto, y a todos los jóvenes de $\mathrm{El}$ Salvador que han compartido generosamente con nosotros sus opiniones.

\section{REFERENCIAS}

1. Ebrahim SH, McKenna MT, Marks JS. Sexual behaviour: related adverse health burden in the United States. Sex Transm Infect. 2005;81(1):38-40.

2. Chesson HW, Blandford JM, Gift TL, Tao GY, Irwin KL. The estimated direct medical cost of sexually transmitted diseases among American youth, 2000. Perspect Sex Reprod Health. 2004;36(1):11-9.

3. Wight D, Parkes A, Strange V, Allen E, Bonell $\mathrm{C}$, Henderson $\mathrm{M}$. The quality of young people's heterosexual relationships: a longitudinal analysis of characteristics shaping subjective experience. Perspect Sex Reprod Health. 2008;40(4):226-37.

4. Louie KS, de Sanjose S, Diaz M, Castellsague X, Herrero R, Meijer CJ, et al. Early age at first sexual intercourse and early pregnancy are risk factors for cervical cancer in developing countries. Br J Cancer. 2009;100(7):1191-97.

5. Ma Q, Ono-Kihara M, Cong L, Xu G, Pan $X$, Zamani $S$, et al. Early initiation of sexual activity: a risk factor for sexually transmitted diseases, HIV infection, and unwanted pregnancy among university students in China. BMC Public Health. 2009;9:111.

6. Dirección de Estadísticas y Censos de El Salvador. Encuesta de Hogares de Propósitos Múltiples 2006. Ciudad Delgado, El Salvador; 2007.

7. Asociación Demográfica Salvadoreña. Encuesta nacional de salud familiar, FESAL 2008. Ciudad Delgado, El Salvador; 2009.

8. Springer AE, Selwyn BJ, Kelder SH. A descriptive study of youth risk behavior in urban and rural secondary school students in El Salvador. BMC Int Health Hum Rights. 2006;6:3.

9. Centeo Monge HL, Cáceres Rodas R. La salud sexual y reproductiva de las jóvenes de 15 a 24 años en El Salvador, un reto para las políticas de salud. Población y Salud en Mesoamérica. 2005;2(2):1-22.

10. Breinbauer C, Maddaleno M. Youth: choices and change. Promoting healthy behaviors in adolescents. Washington D.C.: Pan American Health Organization; 2005.

11. Halperin DT, Steiner MJ, Cassell MM, Green EC, Hearst N, Kirby D, et al. The time has come for common ground on preventing sexual transmission of HIV. Lancet. 2004; 364(9449):1913-5.

12. Alonso A, de Irala J. Strategies in HIV prevention: the A-B-C approach. Lancet. 2004; 364(9439):1033.
13. de Irala J, Alonso A. Changes in sexual behaviours to prevent HIV. Lancet. 2006;368(9549): 1749-50.

14. Blum R. Risk and protective factors affecting adolescent reproductive health in developing countries. Geneva: World Health Organization; 2005.

15. Madkour AS, Farhat T, Halpern CT, Godeau E, Gabhainn SN. Early adolescent sexual initiation as a problem behavior: a comparative study of five nations. J Adolesc Health. 2010;47(4):389-98.

16. Peltzer K. Early sexual debut and associated factors among in-school adolescents in eight African countries. Acta Paediatr. 2010; 99(8):1242-7.

17. Small SA, Luster T. Adolescent sexual activity: an ecological, risk-factor approach. J Marriage Fam. 1994;56:181-92.

18. Salazar LF, Bradley EL, Younge SN, Daluga NA, Crosby RA, Lang DL, et al. Applying ecological perspectives to adolescent sexual health in the United States: rhetoric or reality? Health Educ Res. 2010;25(4):552-62.

19. Kotchick BA, Shaffer A, Forehand R, Miller KS. Adolescent sexual risk behavior: a multisystem perspective. Clin Psychol Rev. 2001; 21(4):493-519.

20. Epstein M, Ward LM. "Always Use Protection": Communication boys receive about sex from parents, peers, and the media. J Youth Adolescence. 2008;37(2):113-26.

21. Wetherill RR, Neal DJ, Fromme K. Parents, peers, and sexual values influence sexual behavior during the transition to college. Arch Sex Behav. 2010;39(3):682-94

22. Henderson M, Butcher I, Wight D, Williamson L, Raab G. What explains between-school differences in rates of sexual experience? BMC Public Health. 2008;8:53.

23. Perrino T, Gonzalez-Soldevilla A, Pantin $\mathrm{H}$, Szapocznik J. The role of families in adolescent HIV prevention: a review. Clin Child Fam Psychol Rev. 2000;3(2):81-96.

24. Velez CN, Rodriguez L, Ungemack JA. Intention to postpone sexual initiation among Puerto Rican female adolescents. P R Health Sci J. 1997;16(2):154-61.

25. Longmore $\mathrm{MA}$, Eng AL, Giordano PC, Manning WD. Parenting and adolescents' sexual initiation. J Marriage Fam. 2009;71: 969-82.

26. Jacobson KC, Crockett LJ. Parental monitoring and adolescent adjustment: an ecological perspective. J Res Adolesc. 2000;10(1):65-97.
27. Schutt-Aine J, Maddaleno Ml. Sexual health and development of adolescents and youth in theAmericas. Programand policyimplications: Pan American Health Organization; 2003.

28. Aspy CB, Vesely SK, Oman RF, Rodine S, Marshall L, McLeroy K. Parental communication and youth sexual behaviour. J Adolesc. 2007;30(3):449-66.

29. McNeely C, Shew ML, Beuhring T, Sieving R, Miller BC, Blum RW. Mothers' influence on the timing of first sex among 14- and 15-yearolds. J Adolesc Health. 2002;31(3):256-65.

30. Walker LR, Rose A, Squire C, Koo HP. Parents' views on sexual debut among pre-teen children in Washington, DC. Sex Education. 2008;8(2):169-85.

31. Dittus PJ, Jaccard J. Adolescents' perceptions of maternal disapproval of sex: relationship to sexual outcomes. J Adolesc Health. 2000; 26(4):268-78.

32. Li N, Boulay M. Individual, familial and extrafamilial factors associated with premarital sex among Bangladeshi male adolescents. Sex Health. 2010;7(4):471-7.

33. Karim AM, Magnani RJ, Morgan GT, Bond KC. Reproductive health risk and protective factors among unmarried youth in Ghana. Int Fam Plan Perspect. 2003;29(1):14-24.

34. Magnani RJ, Seiber EE, Gutierrez EZ, Vereau D. Correlates of sexual activity and condom use among secondary-school students in urban Peru. Stud Fam Plann. 2001;32(1):53-66.

35. Pick de Weiss S, Atkin LC, Gribble JN, Andrade-Palos P. Sex, contraception, and pregnancy among adolescent in Mexico City. Stud Fam Plann. 1991;22(2):74-82.

36. Sanchez N, Grogan-Kaylor A, Castillo M, Caballero G, Delva J. Sexual intercourse among adolescents in Santiago, Chile: a study of individual and parenting factors. Rev Panam Salud Publica. 2010;28(4):267-74

37. Springer AE, Sharma $S$, de Guardado AM Nava FV, Kelder SH. Perceived parental monitoring and health risk behavior among public secondary school students in El Salvador. ScientificWorldJournal. 2006;6:1810-4.

38. de Irala J, Osorio A, López-del Burgo C, Belen VA, de Guzman FO, Calatrava M, et al. Relationships, love and sexuality: what the Filipino teens think and feel. BMC Public Health. 2009:9(282).

39. de Irala J, Osorio A, Carlos S, Ruiz-Canela M, López-del Burgo C. Mean Age of First Sex: Do They Know What We Mean? Arch Sex Behav. 2011;40:853-5. 
40. Lauritsen JL, Swicegood CG. The consistency of self-reported initiation of sexual activity. Fam Plann Perspect. 1997;29(5):215-21.

41. Upchurch DM, Lillard LA, Aneshensel CS, Fang Li N. Inconsistencies in reporting the occurrence and timing of first intercourse among adolescents. J Sex Res. 2002;39(3):197-206.

42. Beguy D, Kabiru CW, Nderu EN, Ngware MW. Inconsistencies in self-reporting of sexual activity among young people in Nairobi, Kenya. J Adolesc Health. 2009;45(6):595-601.

43. Brener ND, Billy JO, Grady WR. Assessment of factors affecting the validity of selfreported health-risk behavior among adolescents: evidence from the scientific literature J Adolesc Health. 2003;33(6):436-57.

44. Biddlecom A, Awusabo-Asare K, Bankole A. Role of parents in adolescent sexual activity and contraceptive use in four African countries. Int Perspect Sex Reprod Health. 2009; 35(2):72-81.

45. Lenciauskiene I, Zaborskis A. The effects of family structure, parent-child relationship and parental monitoring on early sexual behaviour among adolescents in nine European countries. Scand J Public Health. 2008 36(6):607-18.

46. Commendador KA. Parental influences on adolescent decision making and contraceptive use. Pediatr Nurs. 2010;36(3):147-56, 170.

47. Deptula DP, Henry DB, Schoeny ME. How can parents make a difference? Longitudinal associations with adolescent sexual behaviour. J Fam Psychol. 2010;24(6):731-9.

48. Pan American Health Organization. Gender, Health and Development in the Americas. Ba- sic indicators. Washington, DC:Pan American Health Organization; 2005.

49. Huerta-Franco R, Malacara JM. Factors associated with the sexual experiences of underprivileged Mexican adolescents. Adolescence. 1999;34(134):389-401.

50. World Health Organization. Helping parents in developing countries improve adolescents health. Geneva: World Health Organization; 2007.

Manuscrito recibido el 1 de marzo de 2011. Aceptado para publicación, tras revisión, el 19 de julio de 2011.

ABSTRACT Objective. The objective of this study is to verify the influence of messages Salvadorian youth receive about sexuality, affection, and leisure from family, friends, and the media on the initiation of sexual activity.

Family, friends, and other sources of information associated with the initiation of sexual relations by adolescents in El Salvador
Methods. Cross-sectional study based on a representative sample of 2615 students (from 13 to 19 years of age) in El Salvador. A random systematic sampling was used to select 30 schools. Sociodemographic aspects, lifestyles, and sources of information on sexuality and love used by the young people were collected.

Results. The average age of the young people studied was 15 (SD = 1.8). In all, 638 $(24.4 \%)$ of the young people stated that they had had sexual relations. The following factors are associated with a greater probability of having had sexual relations: a perception that siblings ( $\mathrm{OR}=1.8, \mathrm{CI} 95 \%$ : 1.2-2.7) or friends $(\mathrm{OR}=1.7, \mathrm{CI} 95 \%: 1.3-2.2)$ encourage them to have sex. Protective factors were found to be the supervision of parents $(\mathrm{OR}=0.5, \mathrm{CI} 95 \%$ : 0.4-0.7); messages received from friends that encourage abstinence $(\mathrm{OR}=0.7, \mathrm{CI} 95 \%$ : 0.6-1.0) or from siblings $(\mathrm{OR}=0.7, \mathrm{CI} 95 \%$ : 0.5-0.8); and favorable messages related to marriage received from parents $(\mathrm{OR}=0.4, \mathrm{CI} 95 \%$ : 0.3-0.6).

Conclusions. Messages from family and friends are factors that seem to influence the initiation of sexual activity by young people. Sexual health promotion programs in El Salvador should take these factors into account.

Key words Sexual behavior; adolescent behavior; socioeconomic factors; El Salvador. 